\title{
Zero-cycles and rational points on some surfaces over a global function field
}

\section{J.-L. Colliot-Thélène and Sir Peter Swinnerton-Dyer}

Résumé Soit $\mathbf{F}$ un corps fini de caractéristique $p$. Pour une surface lisse sur $\mathbf{F}(t)$ définie par une équation $f+t g=0$, où $f$ et $g$ sont deux formes de degré $d$ sur $\mathbf{F}$ en 4 variables, avec $d$ premier à $p$, nous montrons que l'obstruction de Brauer-Manin au principe de Hasse pour les zéro-cycles de degré 1 est la seule obstruction. Pour $d=3$ (surfaces cubiques), on en déduit le même énoncé pour les points rationnels.

Summary Let $\mathbf{F}$ be a finite field of characteristic $p$. We consider smooth surfaces over $\mathbf{F}(t)$ defined by an equation $f+t g=0$, where $f$ and $g$ are forms of degree $d$ in 4 variables with coefficients in $\mathbf{F}$, with $d$ prime to $p$. We prove : For such surfaces over $\mathbf{F}(t)$, the Brauer-Manin obstruction to the existence of a zero-cycle of degree one is the only obstruction. For $d=3$ (cubic surfaces), this leads to the same result for rational points.

\section{$\S 1$. Introduction}

Let $k$ be a global field.

Study of the case of curves (Cassels, Tate) and of the case of rational surfaces (Colliot-Thélène et Sansuc [CT/S81], where a more precise conjecture is made for rational surfaces) has led to the following conjecture for zero-cycles on arbitrary varieties over global fields (Kato/Saito [K/S86], Saito [S89], Colliot-Thélène [CT93], [CT99]).

Conjecture Let $X$ be a smooth, projective, geometrically integral variety over a global field $k$. If there exists a family $\left\{z_{v}\right\}_{v \in \Omega}$ of local zero-cycles of degree 1 (here $v$ runs through the set $\Omega$ of places of $k$ ) such that for all $A \in \operatorname{Br}(X)$,

$$
\sum_{v \in \Omega} \operatorname{inv}_{v}\left(A\left(z_{v}\right)\right)=0 \in \mathbf{Q} / \mathbf{Z}
$$

holds, then there exists a zero-cycle of degree 1 on $X$. In other words, the Brauer-Manin obstruction to the existence of a zero-cycle of degree 1 on $X$ is the only obstruction.

Over number fields, this conjecture has been established in special cases in work of (alphabetical order, and various combinations) Colliot-Thélène, Frossard, Salberger, Sansuc, Skorobogatov, Swinnerton-Dyer, Wittenberg (see the introduction of [W10]). None of these results applies to smooth surfaces of degree at least 3 in 3 -dimensional projective space - for $d \geq 5$ these surfaces are of general type. In section 2, we establish the conjecture in the special case of a global field $k=\mathbf{F}(t)$ purely transcendental over a finite field $\mathbf{F}$ and of smooth surfaces $X \subset \mathbf{P}_{k}^{3}$ defined by an equation $f+t g=0$, where $f$ and $g$ are two forms of arbitrary degree $d$ over the field $\mathbf{F}$.

According to a conjecture of Colliot-Thélène and Sansuc ([CT/S80]), the Brauer-Manin obstruction to the existence of a rational point on a smooth, geometrically rational surface defined over a global field should be the only obstruction. Such should in particular be the case for smooth cubic surfaces in 3-dimensional projective space $\mathbf{P}_{k}^{3}$. In section 3, we establish the conjecture in the special case of a global field $k=\mathbf{F}(t)$ purely transcendental over a finite field $\mathbf{F}$ and of smooth cubic surfaces $X \subset \mathbf{P}_{k}^{3}$ defined by an equation $f+t g=0$, where $f$ and $g$ are two cubic forms over the field $\mathbf{F}$. Simple though they be, such surfaces may fail to obey the Hasse principle.

\section{$\S 2$. Zero-cycles of degree 1 on surfaces of arbitrary degree}

The following theorem is due to S. Saito [S89]. It says that if a strong integral form of the Tate conjecture on 1-dimensional cycles is true, then the above conjecture holds, at least if we stay away from the characteristic of the field. For an alternate proof of Theorem 1, see [CT99], Prop. 3.2. 
Theorem 1 (Saito) Let $\mathbf{F}$ be a finite field and $C / \mathbf{F}$ a smooth, projective, geometrically integral curve over $\mathbf{F}$. Let $k=\mathbf{F}(C)$ be its function field. Let $\mathcal{X}$ be a smooth, projective, geometrically integral $\mathbf{F}$-variety of dimension $n$ and $f: \mathcal{X} \rightarrow C$ a faithfully flat map whose generic fibre $X / k$ is smooth and geometrically integral.

Assume :

(1) For each prime $l \neq \operatorname{char}(\mathbf{F})$, the cycle map

$$
T_{X}: \mathrm{CH}^{n-1}(\mathcal{X}) \otimes \mathbf{Z}_{l} \rightarrow H_{e ́ t}^{2 n-2}\left(\mathcal{X}, \mathbf{Z}_{l}(n-1)\right)
$$

from the Chow group of dimension 1 cycles on $\mathcal{X}$ to étale cohomology is onto.

(2) There exists a family $\left\{z_{v}\right\}_{v \in \Omega}$ of local zero-cycles of degree 1 (here $v$ runs through the set $\Omega$ of places of $k$ ) such that for all $A \in \operatorname{Br}(X)$,

$$
\sum_{v \in \Omega} \operatorname{inv}_{v}\left(A\left(z_{v}\right)\right)=0 \in \mathbf{Q} / \mathbf{Z} .
$$

Then there exists a zero-cycle on $X$ of degree a power of char $(\mathbf{F})$.

In this statement, $A\left(z_{v}\right)$ is the element of the Brauer group of the local field $k_{v}$ obtained by evaluation of $A$ on the zero-cycle $z_{v}$. The map $\operatorname{inv}_{v}: \operatorname{Br}\left(k_{v}\right) \rightarrow \mathbf{Q} / \mathbf{Z}$ is the local invariant of class field theory.

Here is one case where assumption (1) in the previous theorem is fulfilled.

Theorem 2 Let $\mathbf{F}$ be a finite field and $l$ a prime, $l \neq \operatorname{char}(\mathbf{F})$. For a smooth, projective, geometrically integral threefold $\mathcal{X}$ over $\mathbf{F}$ which is birational to $\mathbf{P}_{F}^{3}$, the cycle map $T_{\mathcal{X}}: C H^{2}(\mathcal{X}) \otimes \mathbf{Z}_{l} \rightarrow H_{\text {ét }}^{4}\left(\mathcal{X}, \mathbf{Z}_{l}(2)\right)$ is onto.

Proof If $\mathcal{X}=\mathbf{P}_{\mathbf{F}}^{3}$, then $C H^{2}(\mathcal{X})=\mathbf{Z}$ and one easily checks that the cycle map

$$
T_{\mathcal{X}}: C H^{2}(\mathcal{X}) \otimes \mathbf{Z}_{l} \rightarrow H_{e t}^{4}\left(\mathcal{X}, \mathbf{Z}_{l}(2)\right)
$$

is simply the identity map $\mathbf{Z}_{l}=\mathbf{Z}_{l}$. Using the standard formulas for the computation of Chow groups and of cohomology for a blow-up along a smooth projective subvariety, as well as the vanishing of Brauer groups of smooth projective curves over a finite field, one shows : For $\mathcal{X}$ a smooth projective threefold, the cokernel of the above cycle map $T_{\mathcal{X}}$ is invariant under blow-up of smooth projective subvarieties on $\mathcal{X}$.

By a result of Abhyankar ([Abh66], Thm. 9.1.6), there exists a smooth projective variety $\mathcal{X}^{\prime}$ which is obtained from $\mathbf{P}_{\mathbf{F}}^{3}$ by a sequence of blow-ups along smooth projective $\mathbf{F}$-subvarieties, and which is equipped with a birational F-morphism $p: \mathcal{X}^{\prime} \rightarrow \mathcal{X}$.

There are push-forward maps $\pi_{*}$ and pull-back maps $\pi^{*}$ both for Chow groups and for étale cohomology, and for the birational map $\pi$ we have $\pi_{*} \circ \pi^{*}=$ id. Moreover these maps are compatible with the cycle class map. Thus the cokernel of $T_{\mathcal{X}}$ is a subgroup of the cokernel of $T_{\mathcal{X}^{\prime}}$, hence is zero. QED

Combining Theorems 1 and 2, we get :

Theorem 3 Let $\mathbf{F}$ be a finite field and $C / \mathbf{F}$ a smooth, projective, geometrically integral curve over F. Let $k=\mathbf{F}(C)$ be its function field. Let $\mathcal{X}$ be a smooth, projective, geometrically integral $\mathbf{F}$-variety of dimension $n$ and $f: \mathcal{X} \rightarrow C$ a faithfully flat map whose generic fibre $X / k$ is smooth and geometrically integral.

Assume :

(1) $\operatorname{dim} \mathcal{X}=3$ and $\mathcal{X}$ is $\mathbf{F}$-rational;

(2) there exists a family $\left\{z_{v}\right\}_{v \in \Omega}$ of local zero-cycles of degree 1 (here $v$ runs through the set $\Omega$ of places of $k$ ) such that for all $A \in \operatorname{Br}(X)$,

$$
\sum_{v \in \Omega} \operatorname{inv}_{v}\left(A\left(z_{v}\right)\right)=0 \in \mathbf{Q} / \mathbf{Z}
$$

Then there exists a zero-cycle on $X$ of degree a power of $\operatorname{char}(\mathbf{F})$. 
We may now prove the main result of this section.

Theorem 4 Let $\mathbf{F}$ be a finite field, let $f, g$ be two nonproportional homogeneous forms in 4 variables, of degree $d$ prime to the characteristic of $\mathbf{F}$. Let $k=\mathbf{F}(t)$. Suppose the $k$-surface $X \subset \mathbf{P}_{k}^{3}$ defined by $f+t g=0$ is smooth. If there is no Brauer-Manin obstruction to the Hasse principle for zero-cycles of degree 1 on $X$, then

(i) there exists a zero-cycle of degree 1 on the $k$-surface $X$;

(ii) there exists a zero-cycle of degree 1 on the $\mathbf{F}$-curve $\Gamma$ defined by $f=g=0$ in $\mathbf{P}_{\mathbf{F}}^{3}$.

Proof Let $\mathcal{X}_{1} \subset \mathbf{P}_{\mathbf{F}}^{3} \times{ }_{F} \mathbf{P}_{\mathbf{F}}^{1}$ be the schematic closure of $X \subset \mathbf{P}_{\mathbf{F}(t)}^{3}$. The $\mathbf{F}$-variety $\mathcal{X}_{1}$ has an affine birational model with equation $\phi(x, y, z)+t \psi(x, y, z)=0$, hence $t$ is determined by $x, y, z$, thus $\mathcal{X}$ is $\mathbf{F}$ birational to $\mathbf{P}_{\mathbf{F}}^{3}$. Since $\mathcal{X}_{1}$ admits a smooth projective model over $\mathbf{F}$, a result of Cossart ([Co92], Théorème, p. 115) shows that there exists a smooth projective threefold $\mathcal{X} / \mathbf{F}$ and an $\mathbf{F}$-birational morphism $\mathcal{X} \rightarrow \mathcal{X}_{1}$ which is an isomorphism over the smooth locus of $\mathcal{X}_{1}$, hence in particular which induces an isomorphism over Spec $\mathbf{F}(t) \subset \mathbf{P}_{\mathbf{F}}^{1}$. That is, the generic fibre of $\mathcal{X} \rightarrow \mathbf{P}_{\mathbf{F}}^{1}$ is $k$-isomorphic to $X / k$.

It remains to combine Theorem 1 and Theorem 2 to prove (i). Statement (ii) follows from (i) as a special application of a result of Colliot-Thélène and Levine ([CT/L09], Théorème 1, p. 217). QED

Remark Theorem 4 is of interest only in the case where the $\mathbf{F}$-curve $\Gamma$ does not contain a geometrically integral component. Otherwise the two statements immediately follow from the Weil estimates for the number of points on geometrically integral curves. These estimates actually provide more : they show that if there exists such a component, then on any field extension $\mathbf{F}^{\prime}$ of $\mathbf{F}$ of high enough degree, there exists an $\mathbf{F}^{\prime}$-point on $\Gamma$, hence for any such field there exists an $\mathbf{F}^{\prime}(t)$-point on the $\mathbf{F}(t)$-surface $X$.

Remark One could try to circumvent the cohomological machinery, i.e. Theorems 1 and 2 . For this, in each of the special cases where there are zero-cycles of degree 1 everywhere locally on $X$ but there is no zero-cycle of degree one on the curve $\Gamma$, one should:

(i) Check that the Brauer group is not trivial, find generators.

(ii) Check that there is a Brauer-Manin obstruction.

Already when the common degree of $f$ and $g$ is 3 , which we shall now more particularly examine, this seems no easy enterprise.

\section{$\S 3$. Rational points on cubic surfaces}

The proof of the following result is independent of the previous results.

Theorem 5 Let $\mathbf{F}$ be a finite field, let $f, g$ be two nonproportional cubic forms over $\mathbf{F}$ in 4 variables. Assume the characteristic of $\mathbf{F}$ is not 3. Let $k=\mathbf{F}(t)$. Suppose the $k$-surface $X \subset \mathbf{P}_{k}^{3}$ defined by $f+t g=0$ is smooth. Let $\Gamma \subset \mathbf{P}_{\mathbf{F}}^{3}$ be the complete intersection curve defined by $f=g=0$. The following conditions are equivalent :

(i) There exists a $k$-rational point on the $k$-variety $X$.

(ii) There exists a zero-cycle of degree 1 on the $k$-variety $X$.

(iii) There exists a zero-cycle of degree 1 on the $\mathbf{F}$-curve $\Gamma$.

(iv) There exists a closed point of degree prime to 3 on the $\mathbf{F}$-curve $\Gamma$.

(v) There exists a closed point of degree a power of 2 on the $\mathbf{F}$-curve $\Gamma$.

Proof That (i) implies (ii) is trivial. That (ii) implies (iii) is a special case of [CT/L09]. Statements (iii) and (iv) are equivalent, since $\Gamma$ is a curve of degree 9 . If (v) holds, then $\Gamma$ has a point in a tower of quadratic extensions of $\mathbf{F}$, hence the cubic surface $X$ has a point in a tower of quadratic extensions of $k$. An extremely well known argument shows that if a cubic surface over a field has a point in a separable quadratic extension of that field, then it has a rational point : the line joining two conjugate points is defined over the ground field, either it is entirely contained in the cubic surface or it meets it in a third, rational point. Iterating this remark, we see that $X$ has a rational point, i.e. (i) holds.

Let us prove that (iii) implies (v). To prove this, one may replace $\mathbf{F}$ by its maximal multiquadratic extension $F$, which we now do. For an odd integer $n$, we let $F_{n} / F$ be the unique field extension of $F$ of degree $n$. 
For $Z / L$ a variety over a field $L$, the index $\operatorname{ind}(Z)=\operatorname{ind}(Z / L)$ is the gcd of the $L$-degrees of closed points on $Z$. The index of an $L$-variety is equal to the index of its reduced $L$-subvariety. The index of an $L$-variety which is a finite union of $L$-varieties is the gcd of the indices of each of them.

Since $F$ has no quadratic or quartic extension, an effective zero-cycle of degree 1,2,4 contains an $F$ rational point, and an effective zero-cycle of degree 3,6 or 9 either contains an $F$-point or has index a multiple of 3 .

If $\Gamma$ contains a geometrically integral component, then $\Gamma(F) \neq \emptyset$ (Weil estimates, see the remark after Theorem 4).

Suppose $\Gamma$ does not contain a geometrically integral component. One then easily checks that the degree 9 curve $\bar{\Gamma}$ can break up only in one of the following ways :

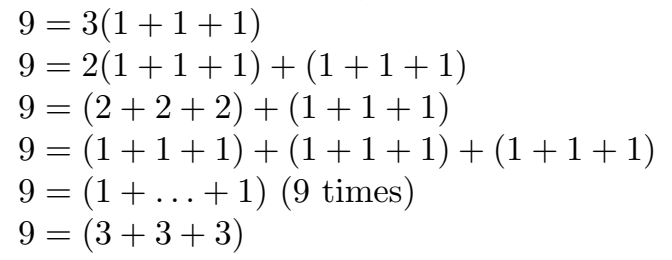

Here $(a+a+a)$ means the sum of 3 conjugate integral curves of degree $a$ over $\bar{F}$.

An integral curve of degree 2 over $\bar{F}$ is a smooth plane conic, contained in a well-defined plane.

An integral curve of degree 3 over $\bar{F}$ is either a plane cubic or a smooth twisted cubic.

Let the integral curve $C \subset \mathbf{P}_{F}^{3}$ break up as $(1+1+1)$. The singular set consists of at most 3 points. Then either $C(F) \neq \emptyset$ or 3 divides ind $(C)$.

Let the integral curve $C \subset \mathbf{P}_{F}^{3}$ break up as $(2+2+2)$. Each conic is defined over $F_{3}$. Two distinct smooth conics on $f=0$ define two distinct planes, hence they intersect in at most 2 geometric points. Such points must already be in $F_{3}$. Thus any closed point in the singular locus of $C$ has degree 1 or 3 . One concludes that either $C(F) \neq \emptyset$ or 3 divides ind $(C)$.

Let the integral curve $\Gamma \subset \mathbf{P}_{F}^{3}$ break up as $(1+\ldots+1)$ (9 times). The 9 lines are defined over $F_{9}$, the degree 9 extension of $F$. So are their intersection points. This implies that any singular closed point on $\Gamma$ has degree a power of 3 . Thus $\Gamma(F) \neq \emptyset$ or 3 divides ind $(\Gamma)$.

Let the integral curve $\Gamma \subset \mathbf{P}_{F}^{3}$ break up as $(3+3+3)$, and assume that this corresponds to a decomposition as three conjugate plane cubics. Each of these is defined over $F_{3}$. The intersection number of two of these cubics is 3 . The points of intersection of two such curves are thus defined over $F_{9}$. We conclude that the singular locus of $\Gamma$ splits over $F_{9}$. This implies that the degree of any closed point in that locus is a power of 3 . Thus either $\Gamma(F) \neq \emptyset$ or 3 divides ind $(\Gamma)$.

Let the curve $\Gamma \subset \mathbf{P}_{F}^{3}$ break up as $(3+3+3)$, and assume that $\Gamma$ breaks up as the sum of three conjugate twisted cubics. The curve $\Gamma$ lies on the smooth cubic surface $X$ over $F(t)$ defined by $f+t g=0$. Each twisted curve is defined over $F_{3}$. Let $\sigma$ be a generator of $\operatorname{Gal}\left(F_{3}(t) / F(t)\right)$. Write $\Gamma=C+\sigma(C)+\sigma^{2} C$ on $X_{F_{3}(t)}$. Using intersection theory on the smooth surface $X_{F_{3}(t)}$, which is invariant under the action of $\operatorname{Gal}\left(F_{3}(t) / F(t)\right)$, and letting $H$ be the class of a plane section, we find $27=(3 H .3 H)=(\Gamma . \Gamma)=3(C . C)+6(C . \sigma(C))$. The curve $C$ is a twisted cubic, hence a smooth curve of genus zero on the smooth cubic surface $X$, whose canonical bundle $K$ is given by $-H$. The formula for the arithmetic genus of a curve on a surface, namely $2\left(p_{a}(C)-1\right)=(C . C)+(C . K)$ gives $(C . C)=1$. This implies $(C . \sigma(C))=4$, hence $\left(\sigma(C) \cdot \sigma^{2}(C)\right)=4$ and $\left(\sigma^{2}(C) . C\right)=4$. Since each of these twisted cubic is defined over $F_{3}$ and since $F_{3}$ has no field extension of degree 2 or 4 , this implies that the points of intersection of any two of these twisted cubics are defined over $F_{9}$. We conclude that the singular locus of $\Gamma$ splits over $F_{9}$. This implies that the degree of any closed point in that locus is a power of 3 . Thus either $\Gamma(F) \neq \emptyset$ or 3 divides ind $(\Gamma)$.

In all cases we have proved : Either $\Gamma(F) \neq \emptyset$ or 3 divides ind $(\Gamma)$.

The assumption ind $(\Gamma)=1$, made in (iii), now implies $\Gamma(F) \neq \emptyset$. QED

Remark If the order $q$ of the finite field $\mathbf{F}$ is large enough and $f+t g=0$ is soluble in $\mathbf{F}(t)$, a variant of the proof for the equivalence of (iv) and (v) shows that $f+t g=0$ has a solution in polynomials of degree at most 5. This raises an interesting general question: are there integers $N(d)$ with the following property? Suppose that $G\left(X_{0}, \ldots, X_{4}, t\right)$ is a polynomial defined over $\mathbf{F}$, homogeneous of degree 3 in the $X_{i}$ and of degree $d$ in $t$; if $G=0$ is soluble in $\mathbf{F}(t)$, then it has a solution in polynomials of degree at most $N(d)$. 
We may now prove :

Theorem 6 Let $\mathbf{F}$ be a finite field, let $f, g$ be two nonproportional cubic forms in 4 variables. Assume the characteristic of $\mathbf{F}$ is not 3 . Let $k=\mathbf{F}(t)$. Suppose the cubic surface $X \subset \mathbf{P}_{k}^{3}$ over $k$ defined by $f+t g=0$ is smooth. If there is no Brauer-Manin obstruction to the Hasse principle for rational points on $X$, then there exists a $k$-rational point on $X$.

Proof Combine Theorem 4 and Theorem 5. QED

Remark Again, it would be nice to avoid the cohomological machinery, i.e. Theorems 1 and 2 . When $X$ has no rational points over $\mathbf{F}(t)$ but points in all the completions of $\mathbf{F}(t)$ one should exhibit an explicit Brauer-Manin obstruction for $X$. For this purpose, it would probably be helpful to use [SD93]. Down to earth computations, which we shall not insert here, have led to the following result. If a smooth cubic surface $X$ given by $f+t g=0$ is a counterexample to the Hasse principle over $\mathbf{F}(t)$, then, after replacing $\mathbf{F}$ by its maximal pro-2-extension $F$, the following holds: When going over to the algebraic closure of $F$, the curve $\Gamma$ in the proof of Theorem 5 breaks up as a sum of 9 conjugate lines, or a sum of three twisted cubics, or a sum of three conjugate conics plus a sum of three coplanar conjugate lines; when using the word "conjugate" we mean that the Galois action is transitive. Only in these three cases may we expect a Brauer-Manin obstruction.

\section{References}

[Abh66] S. Abhyankar, Resolution of Singularities of Embedded Surfaces, Academic Press, New York, 1966.

[CT93] J.-L. Colliot-Thélène, L'arithmétique des zéro-cycles, (exposé aux Journées arithmétiques de Bordeaux, septembre 93), Journal de théorie des nombres de Bordeaux 7 (1995) 51-73.

[CT99] J.-L. Colliot-Thélène, Conjectures de type local-global sur l'image de l'application cycle en cohomologie étale, in Algebraic K-Theory (1997), W. Raskind and C. Weibel ed., Proceedings of Symposia in Pure Mathematics 67, Amer. Math. Soc. (1999) 1-12.

[CT/L09] J.-L. Colliot-Thélène et Marc Levine, Une version du théorème d'Amer et Brumer pour les zéro-cycles, in Quadratic forms, linear algebraic groups, and cohomology (ed. J.-L. Colliot-Thélène, R. S. Garibaldi, R. Sujatha, V. Suresh), Developments in mathematics, Springer-Verlag 18 (2010), 215-223.

[CT/S80] J.-L. Colliot-Thélène et J.-J. Sansuc, La descente sur les variétés rationnelles, in Journées de géométrie algébrique d'Angers (juillet 1979), édité par A.Beauville, Sijthof and Noordhof (1980) $223-237$.

[CT/S81] J.-L. Colliot-Thélène et J.-J. Sansuc, On the Chow groups of certain rational surfaces: a sequel to a paper of S.Bloch, Duke Math. J. 48 (1981) 421-447.

[Co92] V. Cossart, Modèle projectif régulier et désingularisation, Math. Annalen 293 (1992) $115-121$.

[K/S86] K. Kato et S. Saito, Global class field theory of arithmetic schemes, Contemporary math. 55, vol. 1 (1986) 255-331.

[S89] S. Saito, Some observations on motivic cohomology of arithmetic schemes, Invent. math. 98 (1989) 371-404.

[SD93] Sir Peter Swinnerton-Dyer, The Brauer group of cubic surfaces, Math. Proc. Camb. Phil. Soc. 113 (1993) 449-460.

[W10] O. Wittenberg, Zéro-cycles sur les fibrations au-dessus d'une courbe de genre quelconque, http://arxiv.org/abs/1010.1883 
J.-L. Colliot-Thélène,

C.N.R.S.,

Mathématiques, Bâtiment 425,

Université Paris-Sud

F-91405 Orsay

France

jlct@math.u-psud.fr

Sir Peter Swinnerton-Dyer,

Departement of Pure Mathematics and Mathematical Statistics, Centre for Mathematical Sciences,

Wilberforce Road,

Cambridge CB3 0WA

England

H.P.F.Swinnerton-Dyer@dpmms.cam.ac.uk 